\title{
Accessibility of Website for Visually Challenged: Combined Tree Structure and XML Metadata
}

\author{
Young Lee, Victoria Christy Sathya Rajasekar, and Prawanth Reddy Kasula \\ Department of Electrical Engineering and Computer Science, \\ Texas A\&M University Kingsville, USA \\ \{young.lee@tamuk.edu, victoriachristysathya@gmail.com, prawanth@gmail.com\}
}

\begin{abstract}
This paper presents two different approaches to provide an efficient multimedia interface for the visually challenged people. The first approach is the tree structured web contents, which integrates many different web pages into a single page. The second approach is the XML metadata for dynamically changing multimedia contents. This paper demonstrates how this combined approach of tree structure and metadata can help visually challenged users accessing multimedia data on the web.
\end{abstract}

\section{INTRODUCTION}

One of the major problems for the visually challenged in accessing the web page is Navigation. Considerable advancements have been made so far to enable navigation in a simple and user-friendly way. In this research tree structure has been implemented to avoid this problem.

Another major problem is to access the dynamically changing multimedia contents. Generally, screen readers like JAWS, Window-Eyes, IBM's AI Browser, etc, are used by the blind people to browse the internet. The screen reader finds it difficult to recognize the rapid changes in the multimedia contents and thus the blind and visually impaired will not receive all the information [1].

Two proposed techniques have been integrated in this research to overcome these problems. In the tree structure, there is a single integrated web page in which the structure of the entire web pages in a website is displayed in a hierarchy [2]. For accessing the multimedia contents of webpage, external metadata is used to describe the dynamically changing multimedia contents of the web page.

\section{RELATED WORKS}

Some of the advancements for the visually disabled people are Screen readers, Braille, Note takers, and Optical Character Recognition. The screen reader produces voice output for text displayed on the computer screen as well as keystrokes entered on the keyboard. Thus the text given in the website can be converted into speech and the user can access it. Certain image descriptions are given as alternate text so that the screen reader converts it into speech [3]. In case of multimedia contents, the screen reader instructs the user to navigate to the audio/video content. But once the audio starts playing it becomes difficult for the users to listen to the screen reader. Research has been done to sidestep such problems by providing required shortcut keys [2].

'Window-Eyes' is screen reader software that reads out the contents shown in the computer and helps the blinds use the programs. Some programs like, Eudora, Ease reader, etc, are specially designed for the visually impaired for emailing and for reading e-books. IBM has developed AI browser to allow users to access the Internet. Many such improvements have been made.

The major problem discussed earlier is navigating through the web. This problem has been analyzed and required measures are suggested. Some of them are, using JAWS with IE to directly jump from the current position to the next hyperlink element, to the next interactive control, generating alternative views of the webpage, and creating list of hyperlinks in the page. But all these are not systematic and well integrated. So an efficient way of navigation is to provide a single integrated webpage view which can be dynamically and interactively varied based on the page structure [2]. 


\section{APPROACH}

There are two techniques integrated together in this research, (i) Implementation of tree structure in a website to enable simple navigation and (ii) Creation of external metadata to describe the dynamically changing multimedia content. These two approaches are discussed in detail in following sections.

\section{A. Tree Structure}

A website can be completely accessed by a visually disabled user only if the necessary contents of the pages are easy to navigate. The user should be able to browse the websites without any difficulties. There is no benefit in designing a website that is not easily accessible by the user.

A tree structure in a website integrates all linear web pages into a single page. This has been implemented in this research by using Microsoft .net framework with Java script. Physically there is only one web page that is a single integrated page, consisting of two frames. One contains the tree structure of the web pages and the other frame displays the web page that is being selected from the tree. Since the hierarchy of the website is known before, the user can directly open a web page and move to different levels. The users can create their own view of the web page by expanding or collapsing the nodes of the tree structure. Expanding nodes result in displaying the page in frame 2 and collapsing the nodes hides the pages. This demonstrates user friendliness.

\section{B. Sample Tree Structure}

In this sample tree structure the single integrated page consists of two frames, where the first frame contains the actual tree structure. If a node is selected in the first frame, that particular page will open in the second frame, as shown in Fig. 1.

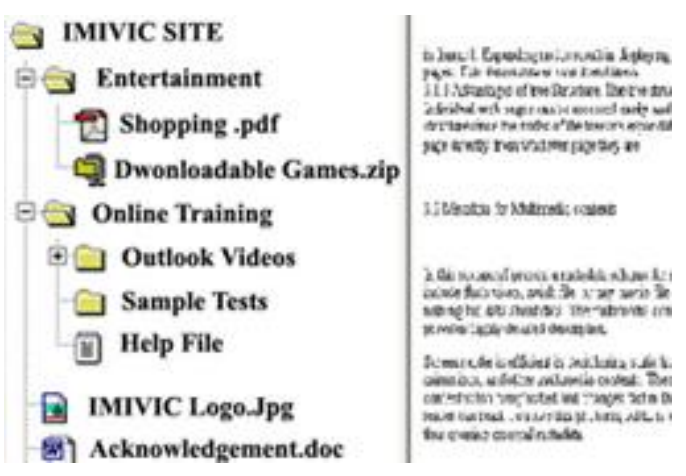

Fig. 1. Sample Tree Structure
The tree structure is used in websites due to several advantages. (i) Individual web pages can be accessed easily and quickly, (ii) Users can create their own view of the tree structure since the nodes of the tree are expandable and collapsible, (iii) Users can return to the home page directly from whatever page they are.

\section{Metadata for Multimedia contents}

In this research a metadata scheme for multimedia content description is provided. The multimedia contents include flash video, swish file, or any image/movie file that changes dynamically in a web page. Metadata is data about data. The multimedia content description files which are the external metadata are created using XML which provides highly detailed description [4].

Screen reader is efficient in deciphering static html pages but not the dynamic contents like flash animations, and other multimedia contents. These cannot be rendered as speech because the multimedia content is too complicated and changes rapidly in the web page and has no alternate text that the screen reader can read. To solve this problem, XML is used to describe the dynamically changing web content, thus creating external metadata.

\section{EVALUATION}

The objectives of this empirical study are to investigate if tree structure and XML metadata help visually challenged to navigate website. A sample multimedia content website with tree structure hierarchy was created, so that a Screen reader can read the description behind the content when the control is over the particular multimedia content.

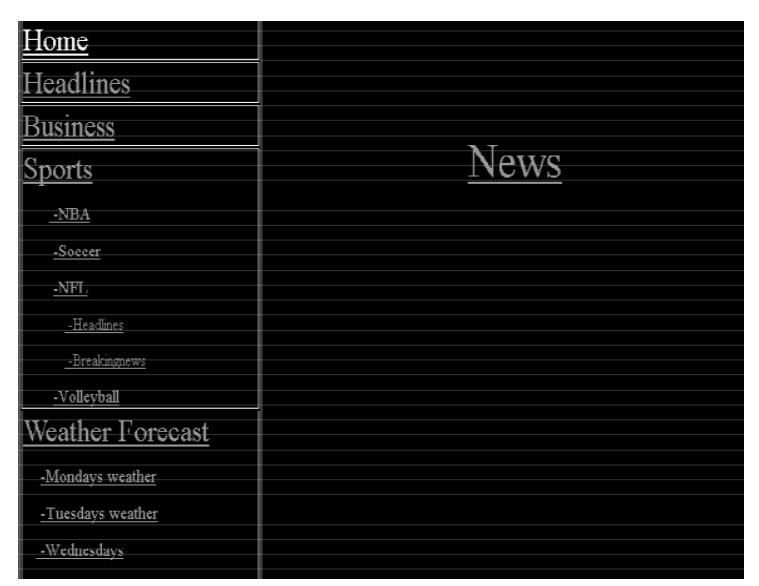

Fig. 2. Website with Tree structure 
In figure 2 the root node is divided into five different nodes namely home, headlines, business, sports and weather forecast. The sports node is further divided into four sub nodes namely NBA, soccer, NFL and volleyball. The node NFL is further divided into two sub nodes namely headlines and breaking news. Thus it forms a tree structure, which can be opened and collapsed.

A sample website similar to the above website was created but the structure was not in a tree hierarchy. And there was no description for the multimedia contents.

Table 1: Test Results

\begin{tabular}{|c|c|c|c|c|}
\hline Volunteer no: & $\begin{array}{l}\text { Time taken in } \\
\text { Tree Structure }\end{array}$ & $\begin{array}{l}\text { Time taken in } \\
\text { Linear } \\
\text { Structure }\end{array}$ & (D) & $\begin{array}{l}\text { Signed } \\
\text { Rank }\end{array}$ \\
\hline 1 & 185 & 220 & 35 & 14 \\
\hline 2 & 163 & 222 & 59 & 21 \\
\hline 3 & 121 & 182 & 61 & 22 \\
\hline 4 & 150 & 163 & 13 & 4 \\
\hline 5 & 202 & 266 & 64 & 23 \\
\hline 6 & 192 & 318 & 126 & 29 \\
\hline 7 & 235 & 241 & 6 & 2 \\
\hline 8 & 149 & 176 & 27 & 9 \\
\hline 9 & 155 & 183 & 28 & 10.5 \\
\hline 10 & 220 & 223 & 3 & 1 \\
\hline 11 & 230 & 239 & 9 & 3 \\
\hline 12 & 290 & 330 & 40 & 15 \\
\hline 13 & 113 & 222 & 109 & 27 \\
\hline 14 & 182 & 236 & 54 & 19 \\
\hline 15 & 210 & 286 & 76 & 24 \\
\hline 16 & 242 & 160 & -82 & -25 \\
\hline 17 & 300 & 274 & -26 & -8 \\
\hline 18 & 226 & 344 & 118 & 28 \\
\hline 19 & 252 & 293 & 41 & 16 \\
\hline 20 & 204 & 176 & -28 & -10.5 \\
\hline 21 & 230 & 259 & 29 & 12 \\
\hline 22 & 200 & 243 & 43 & 17 \\
\hline 23 & 170 & 302 & 132 & 30 \\
\hline 24 & 165 & 198 & 33 & 13 \\
\hline 25 & 132 & 176 & 44 & 18 \\
\hline 26 & 183 & 199 & 16 & 6.5 \\
\hline 27 & 180 & 235 & 55 & 20 \\
\hline 28 & 175 & 189 & 14 & 5 \\
\hline 29 & 195 & 106 & -89 & -26 \\
\hline 30 & 150 & 166 & 16 & 6.5 \\
\hline
\end{tabular}

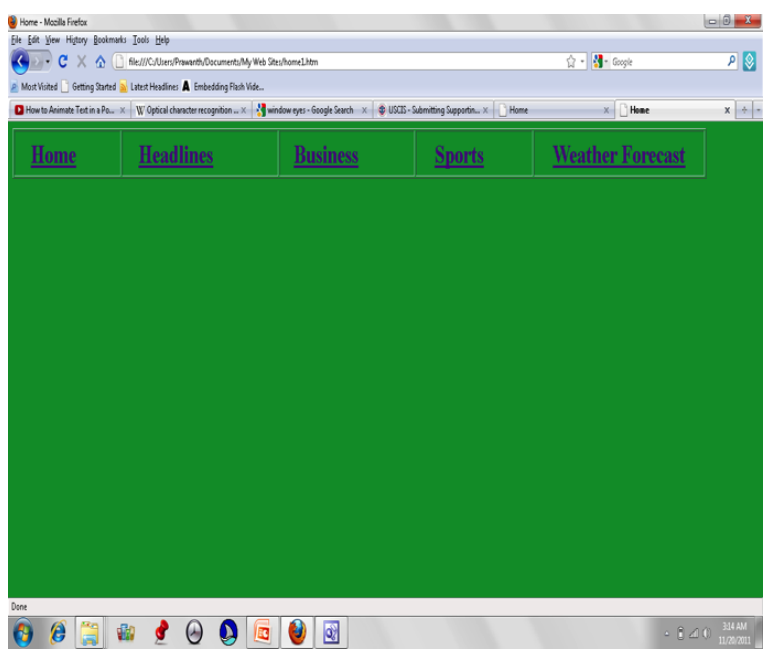

Fig. 3. Website with Linear structure.

Figure 3 is a simple linear website which has five links similar to that of figure 2, but there is no proper navigation from one link to the other. The user has to come back to the home page to navigate from one link to the other.

Two test cases have been assigned in which, the first test case is the decision maker for the navigation time and the second test case decides about the understandability of the multimedia content between both the structures.

Hypothesis: Tree structure has positively improved accessibility for visually challenged.

Test Case 1: To listen to the Sunday's weather report at weather forecast and then listen to the volleyball news at sports.

After setting up the testing environment, 60 blind folded volunteers were randomly divided into two groups of 30 each, where the first group were to follow the test case with tree structure and the second group were to follow the test case with linear structure.

\section{Test Result}

According to the Wilcoxon Signed Rank Test for two samples, the null hypothesis and the alternate hypothesis were firstly defined as

$\mathrm{H}_{0}$ : Tree Structure has no improvement in accessibility $\mathrm{H}_{\mathrm{a}}$ : Tree Structure has improved the accessibility Difference $(\mathrm{D})=($ Time taken in Tree Structure $)-($ Time taken in Linear Structure) 
Ranking is given ignoring the sign for all the values of $\mathrm{D}$, then the sign is placed back on to the rank as shown in Table 1. The Test Statistic T is determined as

$\mathrm{T}=$ Maximum of $\mathrm{T}-$ and $\mathrm{T}+$

where

$\mathrm{T}-\mathrm{=}$ Sum of all ranks with negative sign

$\mathrm{T}+=$ Sum of all ranks with positive sign.

From the test result (Table 1) the critical region is 106 , as it is recorded as the worst case among all the volunteers. If $\mathrm{T}$ is greater than 106 then the value lies in the critical region and null hypothesis can be rejected.

$\mathrm{T}$ - is -69.5 and $\mathrm{T}+$ is 395.5 , therefore $\mathrm{T}$ is 395.5 . The null hypothesis $\mathrm{H}_{0}$ can be rejected with respect to the alternate hypothesis $\mathrm{H}_{\mathrm{a}}$. According to the Wilcoxon Signed Rank Test, Tree structure has positively improved the accessibility of visually challenged.

Test Case 2: To listen to the headlines at headlines.

30 blindfolded volunteers were made to listen to the headlines at headlines with both tree structure and linear structure and the feedback was recorded that was drawn on a bar diagram as shown figure 4 .

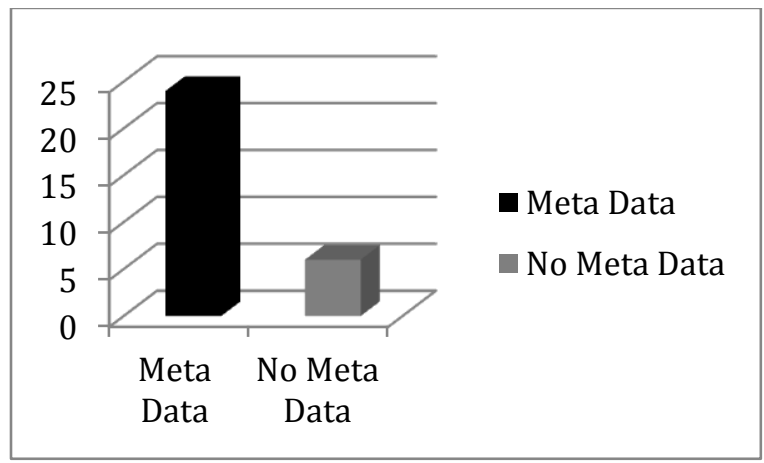

Fig. 4. Bar diagram for Meta data

In figure 4,24 out of 30 volunteers supported metadata and the remaining 6 volunteers did not find any difference between the one with description and the one without description.

\section{CONCLUSION}

This paper presented that the combination of the tree structure and metadata techniques gives efficient navigation and efficient access to the multimedia contents by the visually challenged.

Tests results concluded that tree structure is faster than the linear structure for web site navigation and external metadata for multimedia contents of the web pages provides easy understanding.

The tree structure web pages are integrated in a single page, web pages from any different levels can be accessed directly. The metadata description helps visually. Thus tree structure and metadata for multimedia web sites provide the visually challenged users with a better web browsing environment.

\section{REFERENCES}

[1] Hisashi Miyashita, Daisuke Sato, Making Multimedia Content Accessible for Screen Reader Users, IBM Research, Tokyo Research Laboratory, $16^{\text {th }}$ international World Wide Web conference, 2007.

[2] Esmond Walshe, Barry McMullin, Research Institute for Networks and Communications Engineering (RINCE), Accessing Web Based Documents Through a Tree Structural Interface, In Proceedings of ICCHP 2004.

[3] Kristy Williamson, Steve Wright, Don Schauder, and Amanda Bow, The Internet for the Blind Visually Impaired, JCMC 7 (1), October 2001.

[4] Geoff Freed, The Web Access Project - Access to Multimedia on the Web, National Center for Accessible Media, 1997 


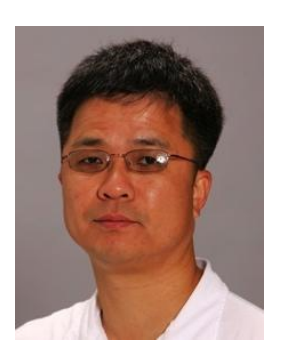

Young Lee is an assistant professor in the department of electrical engineering and computer science at Texas A\&M University-Kingsville. $\mathrm{He}$ received his Ph.D. in computer science and software engineering from Auburn University.

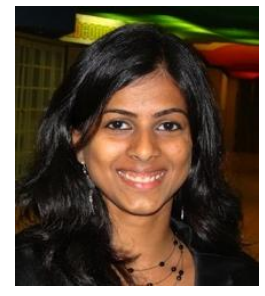

Victoria Christy Sathya Rajasekar is a senior software consultant st Capgemini. She received her M.S. degree in electrical engineering and computer science at Texas A\&M University-Kingsville.

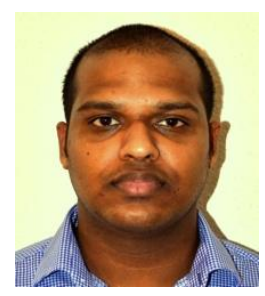

Prawanth Reddy Kasula received his M.S. degree in electrical engineering and computer science at Texas A\&M University-Kingsville. 